\title{
GEOMETRIC ASPECTS OF REDUCTION OF ORDER
}

\author{
JAMES SHERRING AND GEOFF PRINCE
}

\begin{abstract}
Using the differential geometry of vectorfields and forms we reinterpret and extend the traditional idea of an integrating factor for a first order differential equation with symmetry. In particular, we provide a simple and manifestly geometric approach to reduction of order via symmetry for ordinary differential equations which largely obviates the necessity for canonical coordinates and the associated quotient manifolds. In so doing, some new results which generalise the class of Lie group actions which can be used to solve ordinary differential equations are developed.
\end{abstract}

\section{INTRODUCTION}

"Reduction of order via symmetry" for ordinary differential equations has a more or less standard meaning. It involves, in any particular case, the introduction of canonical coordinates tailored to the symmetry group which then enables an explicit presentation of a reduced differential equation on an appropriate quotient manifold. In the case of a single first order equation there is an alternative: an integrating factor can be constructed from the symmetry and this converts the equation to an exact one. Common wisdom has it that the technique cannot be used except in this case.

It is our aim here to provide the generalisation of the integrating factor approach. To achieve this we cast the first order result into a geometric form using differential forms and geometric notions of symmetry and Frobenius integrability; the path to the general result then becomes clear. A coordinate-free approach has both aesthetic and practical advantages. First of all, the final theory, while local like its canonical coordinate counterpart, does not have to be concerned with the paraphernalia of coordinate charts to nearly the same extent. Moreover, the theory in geometric notation is as straightforward as its appearance. Secondly, most specific calculations can be performed in the coordinates in which the problem was originally posed, a big advantage for al-

Received by the editors September 4, 1990.

1991 Mathematics Subject Classification. Primary 58F35, 58A17; Secondary 58A15.

Key words and phrases. Reduction of order, symmetry of a differential equation, symmetry of a Pfaffian system.

We owe a debt of gratitude to Grant Cairns who has been a tireless and constructive critic, also Willy Sarlet, Mike Crampin and Sue Godfrey for many useful discussions. James Sherring acknowledges a Commonwealth Postgraduate Research Award and the hospitality of the Department of Mathematics, Statistics and Computing Science at the University of New England, Armidale. Geoff Prince acknowledges the hospitality of the Department of Mathematical Physics at University College, Dublin and the Instituut voor Theoretische Mechanica at Rijksuniversiteit, Gent. 
gebraic computing. And the geometric theory considerably broadens the class of one-parameter local Lie group actions that can be involved in the process of integrating systems of ordinary differential equations of arbitrary order.

In $\S$ II we present Lie's integrating factor result and give it a geometric formulation that is fairly easily generalised. In $\S$ III we look at second order equations and present our "integrating factor" result, namely the construction of first integrals and hence solutions from generators of local one-parameter groups and a certain 2-form which characterises the differential equation. Basic technical definitions appear in the fourth section before we develop some general results about Frobenius integrable forms and their symmetries. You may find it helpful to read these definitions at the outset. In the fifth section we return to differential equations and use the results of $\S I V$ to obtain a very general result about the reduction of an arbitrary system of ordinary differential equations. We show that the classical theorem concerning reduction of order by solvable subalgebras of point symmetries is a special case of this result. Finally we present some connections with the classical theory of canonical coordinates and quotients.

We have purposely begun this paper with the familiar cases of first and second order equations; we take the view that the results will be more accessible if they are presented from the bottom up. It is our hope that you will come away with a new and usable technique for solving particular problems as well as an understanding of our attempts at a formal theory.

Finally, we cite the following sources: Olver [1] is a seminal work on Lie groups and differential equations, Vaisman [2] contains the material on foliated exterior derivatives that we use, we have used the notation and terminology on the Frobenius theorem from Crampin and Pirani [3] and last, and probably most importantly, Cartan [4-5] has inspired many of our ideas. It is a great pity that these works by one of the founders of the theory have not appeared in English.

\section{FIRST ORDER ORDINARY DIFFERENTIAL EQUATIONS}

The correspondence between symmetries and integrating factors for explicit first order ordinary differential equations is well known. In particular, consider the first order equation

$$
\frac{d y}{d x}=F(x, y)
$$

defined on some open subset $U$ of $\mathbf{R}^{2}$, where $F$ is nowhere zero. This can be written as the total differential equation

$$
d y-F d x=0 \text {. }
$$

Then suppose this equation has a nowhere zero symmetry

$$
V=P \frac{\partial}{\partial x}+Q \frac{\partial}{\partial y}
$$

in the sense of Lie, with $P$ and $Q$ functions on $U$. That is, $V$ is a vectorfield on $U$ which is the infinitesimal generator of a one-parameter transformation group on $U$ which takes solutions of (2-1) into other solutions. 
The well-known result is that an integrating factor making the total differential equation exact is given by

$$
I=\frac{1}{Q-P F} \text {. }
$$

The generalisation of this result to higher order cases is clumsy without some differential geometry, so it is appropriate to introduce it here. We work as usual on some smooth, constant dimensional, second countable, Hausdorff manifold, and assume all functions, vectorfields and differential forms which we use are also smooth. The total differential equation becomes a 1-form

$$
\omega=d y-F d x
$$

which is a cross section of $T^{*} U$, that is, $\omega \in \bigwedge^{1} U$. The characteristic vectorfield of $\omega$ is

$$
\Gamma=\frac{\partial}{\partial x}+F \frac{\partial}{\partial y}
$$

this is a vectorfield tangent to the solution curves, and the normalisation condition that $\Gamma(x)=1$ is equivalent to requiring that the solution curves be parameterised by $x$. The Pfaffian equation " $\omega=0$ " (which is interpreted as $\omega=0$ on solutions) is then given meaning as determining the congruence of curves on $U$ whose tangent vectorfield $\Gamma$ satisfies $\Gamma\lrcorner \omega=0$. The infinitesimal criterion for $V$ to be a symmetry of (2-1) in terms of the Lie derivative is then $\mathscr{L}_{V} \Gamma=\lambda \Gamma$ for some $\lambda \in \bigwedge^{0} U$ (functions or 0 -forms on $U$ ) or dually that $\mathscr{L}_{V} \omega=\bar{\lambda} \omega$. An integrating factor for $\omega$ in this setting is a nowhere zero multiplying function $I$ so that the exterior derivative $d(I \omega)$ is zero. Geometrically, $I$ is given by $(V\lrcorner \omega)^{-1}$ (or equivalently $\left(i_{V} \omega\right)^{-1}$ or $\langle V, \omega\rangle^{-1}$ ) so that provided $V\lrcorner \omega$ is nonzero everywhere on some open $U^{\prime} \subseteq U$, then

$$
d \frac{\omega}{V\lrcorner \omega}=0 \text {. }
$$

By virtue of the converse of the Poincare Lemma which states a closed form is exact in any starlike region, we have the representation

$$
\frac{\omega}{V\lrcorner \omega}=d f
$$

for some locally defined $f$. This function $f$ is known as a differential invariant or first integral of the differential equation and has constant value on any particular solution curve on any starlike open subset of $U^{\prime}$. For example, take the very simple equation

$$
\frac{d y}{d x}=\frac{y}{x}
$$

defined on $U=\left\{(x, y) \in \mathbf{R}^{2}: x \neq 0\right\}$ with the Pfaffian 1-form $\omega=d y-$ $y / x d x$ on $U$, and consider the infinitesimal generator

$$
V=x \frac{\partial}{\partial y}-y \frac{\partial}{\partial x}
$$

of rotations about the origin. Now $\mathscr{L}_{V} \omega=-\frac{y}{x} \omega$ so $V$ is a symmetry of (2-2) and so provides an integrating factor. Thus the expression

$$
\frac{\omega}{V\lrcorner \omega}=\frac{x d y-y d x}{x^{2}+y^{2}}
$$


is locally exact, and upon integration we find

$$
\frac{\omega}{V\lrcorner \omega}=d \theta
$$

where $\theta$ is the polar angle $\operatorname{artan}(y / x)$. We may choose our branch of $\operatorname{artan}$ consistently so that $\theta$ is well defined on some subset of $U$, but were we to try to integrate

$$
\frac{x d y-y d x}{x^{2}+y^{2}}
$$

on the whole of $\mathbf{R}^{2} \backslash\{0\}$ (which is not starlike) then we would have the usual problems with defining $\theta$. The first integral $\theta$ implies that the general solution to $(2-2)$ is $y=m x$ on $U$ for $m \in \mathbf{R}$.

We conclude this section with a proof of the integrating factor result in its most general formulation.

Theorem 2.1. Let $\theta$ be a Frobenius integrable 1-form which is nowhere zero on some open subset $U$ of a manifold $M$. Then $I$ is an integrating factor for $\theta$ if and only if $I=(X\lrcorner \theta)^{-1}$ for some symmetry vectorfield $X$ on $U$ with $\left.X\right\lrcorner \theta \neq 0$ on $U$.

Proof. Suppose that $\mathscr{L}_{X} \theta=\beta \theta$ (this is what we mean by symmetry in this context). Then we have on $U$

$$
\begin{aligned}
(X\lrcorner \theta)^{2} d \frac{\theta}{X\lrcorner \theta} & =-d(X\lrcorner \theta) \wedge \theta+(X\lrcorner \theta) d \theta \\
& \left.\left.=\left(-\mathscr{L}_{X} \theta+X\right\lrcorner d \theta\right) \wedge \theta+(X\lrcorner \theta\right) d \theta \\
& =-\beta \theta \wedge \theta+X\lrcorner(d \theta \wedge \theta) \\
& =0
\end{aligned}
$$

as $\theta \wedge \theta=0$ by degree and $d \theta \wedge \theta=0$ by Frobenius integrability. Conversely, suppose $I$ is an integrating factor for $\theta$ so $d(I \theta)=0$. Then there exists a nonunique $X$ on $U$ such that $I=(X\lrcorner \theta)^{-1}$ with $\left.X\right\lrcorner \theta \neq 0$ everywhere by virtue of $\theta$ and $I$ being nowhere zero. Using the relationship between Lie derivative, exterior derivative and the interior product we have

$$
\left.\left.\mathscr{L}_{X}(I \theta)=X\right\lrcorner d(I \theta)+d(X\lrcorner I \theta\right)=0,
$$

and combined with the product rule for the Lie derivative

$$
\mathscr{L}_{X}(I \theta)=X(I) \theta+I \mathscr{L}_{X} \theta,
$$

the symmetry condition is thus satisfied with

$$
\mathscr{L}_{X} \theta=-\frac{X(I)}{I} \theta \text {. }
$$

Note that the Frobenius integrability of a 1 -form $\theta$ implies the existence of some 1-form $\alpha$ such that $d \theta=\alpha \wedge \theta$. So if $V$ is a vectorfield such that $V\lrcorner \theta=0$ then $\left.\mathscr{L}_{V} \theta=(V\lrcorner \alpha\right) \theta$ and so $V$ is automatically a trivial symmetry of $\theta$. Every Frobenius integrable 1 -form locally has an integrating factor, and by Theorem 2.1 it thus has a symmetry $X$, not in its kernel. For any vectorfield $Y$ nowhere in the kernel of $\theta$ we can find some factor $J$ such that $J Y$ differs from $X$ only by some vectorfield in the kernel of $\theta$, so $J Y$ is necessarily a symmetry of $\theta$. The factor $J$ is called a symmetrising factor. The problem of 
finding an integrating factor for $\theta$ is thus equivalent to finding a symmetrising factor for some $Y$ nowhere in the kernel of $\theta$. This emphasizes the point that it is formally no easier to find all generalised symmetries of a differential equation than it is to solve the equation, however, geometrical insight may provide us with symmetries rather than solutions.

\section{SECOND ORDER ORDINARY DIFFERENTIAL EQUATIONS}

When we approach equations of order greater than one, it is no longer possible to solve the equation (just using symmetry techniques) with the aid of a single symmetry. In the case of a variational problem and a variational symmetry we may use Noether's theorem to construct a first integral, but otherwise the best we can do is to reduce the order of the equation by one. The use of canonical coordinates to effect this reduction is well understood, see Olver [1] for example. However, the generalisation of the integrating factor method is not well known and the presentation of the reduction process outlined here is new.

If we consider an explicit second order differential equation

$$
\frac{d^{2} y}{d x^{2}}=F\left(x, y, \frac{d y}{d x}\right)
$$

then the geometrical setting we use is some open subset $U$ of the first order jet bundle $J^{1}(\mathbf{R}, \mathbf{R})$ with coordinates $x, y$ and $p=\frac{d y}{d x}$. The characteristic vectorfield for (3-1), or the tangent vectorfield to the solution curves lifted from $J^{0}(\mathbf{R}, \mathbf{R})$ to $U$, is $\Gamma=\frac{\partial}{\partial x}+p \frac{\partial}{\partial y}+F \frac{\partial}{\partial p}$, and this has a dual distribution spanned by the contact form $\theta=d y-p d x$ and the force form $\phi=d p-F d x$. The 2-form $\Omega=\theta \wedge \phi$ on $U$ is then a characterising form for (3-1).

The problem is to find two linearly independent combinations of $\theta$ and $\phi$ over $\bigwedge^{0} U$ which are closed 1 -forms, thus providing two first integrals and an implicit general solution. (Note that we are now regarding the space $\wedge^{1} U$ of 1 -forms (which, remember, are fields) as a module, denoted $\mathfrak{X}^{*}(U)$, over the smooth functions $\wedge^{0} U$, this is an idea we will use throughout this paper. In particular, if we require a set of vectorfields or forms to be linearly dependent over $\Lambda^{0} U$ then this is equivalent to requiring the vectors (or forms) of those fields to be pointwise linearly dependent everywhere.) However, if we can manage to find linear combinations which are Frobenius integrable rather than closed then we have reduced the problem to the first order case. It is elementary to see that any such combination can be expressed as $V\lrcorner \Omega$ for some vectorfield $V$ on $U$, and the following results tell us that in particular we can use symmetry vectorfields, that is vectorfields $V$ with $\mathscr{L}_{V} \Gamma=\lambda \Gamma$, or equivalently, $\mathscr{L}_{V} \Omega=\bar{\lambda} \Omega$.

Proposition 3.1. If $V$ is a vectorfield whose span with $\Gamma$ is two dimensional on $U$ then the 1-form $V\lrcorner \Omega$ is Frobenius integrable if and only if $V$ and $\Gamma$ are in involution.

Proof. The kernel of $V\lrcorner \Omega$ is spanned by $V$ and $\Gamma$, and a form is Frobenius integrable if and only if its kernel is of maximal dimension and in involution.

Corollary 3.2. If $V$ is a nontrivial symmetry of (3-1) then $V\lrcorner \Omega$ is Frobenius integrable. 
Corollary 3.3. If $V$ and $W$ are symmetries of (3-1) which commute and whose span with $\Gamma$ is three dimensional then the two forms

$$
\frac{V\lrcorner \Omega}{W\lrcorner V\lrcorner \Omega} \quad \text { and } \quad \frac{W\lrcorner \Omega}{V\lrcorner W\lrcorner \Omega}
$$

are closed and locally provide a complete set of two functionally independent first integrals.

Proof. We have that $V\lrcorner \Omega$ is Frobenius integrable, and to show that $W$ is a symmetry of $V\lrcorner \Omega$ it suffices to note that $W$ is a symmetry of $V$ and $\Gamma$ and hence of the distribution spanned by $V$ and $\Gamma$, which is the kernel of $V\lrcorner \Omega$. Thus $(W\lrcorner V\lrcorner \Omega)^{-1}$ provides an integrating factor for $\left.V\right\lrcorner \Omega$ and likewise $(V\lrcorner W\lrcorner \Omega)^{-1}$ provides an integrating factor for $\left.W\right\lrcorner \Omega$. Now the two closed forms are linearly independent over $\Lambda^{0} U$ and so their integration yields functionally independent first integrals on $U$.

The two first integrals of this corollary, together with $x$, provide a coordinate chart which straightens out the integral curves of $\Gamma$.

The following corollary shows how a symmetry and an "almost symmetry" provide us with one closed form $\omega^{1}$ and a second form $\omega^{2}$ which is closed modulo $\omega^{1}$, that is $d \omega^{2}=0 \bmod \omega^{1}$ or locally $\omega^{1}=d \gamma^{1}$ and $\omega^{2}=d \gamma^{2}+$ $\gamma^{0} d \gamma^{1}$. (The integration of a form closed modulo other closed forms is as easy as integrating a closed form. In fact it involves one less quadrature; see Sherring [6] for a computer algebra program.)

Corollary 3.4. If $V$ is a symmetry of (3-1), $W$ is a symmetry of $\operatorname{span}(\{V, \Gamma\})$ and $V, W$ and $\Gamma$ are linearly independent everywhere then

$$
\omega^{1}=\frac{V\lrcorner \Omega}{W\lrcorner V\lrcorner \Omega}
$$

is closed, while

$$
\omega^{2}=\frac{W\lrcorner \Omega}{V\lrcorner W\lrcorner \Omega}
$$

is closed modulo $\omega^{1}$. These two forms locally provide a complete set of two functionally independent first integrals, $\omega^{1}=d \gamma^{1}$ and $\omega^{2}=d \gamma^{2}-W\left(\gamma^{2}\right) d \gamma^{1}$. By putting $\widehat{W}=W-W\left(\gamma^{2}\right) V$ we then have the two commuting symmetries $V$ and $\widehat{W}$ which provide $\gamma^{1}$ and $\gamma^{2}$ via Corollary 3.3 .

Proof. That $\omega^{1}$ is closed follows from the preceding argument, and suppose that locally $\omega^{1}=d \gamma^{1}$. To see that $\omega^{2}$ is closed modulo $\omega^{1}$ we consider the foliation $\mathscr{F}$ of $M$ by the distribution $\operatorname{ker} \omega^{1}$ (meaning that the leaves of the foliation are given by the integral manifolds with $\operatorname{ker} \omega^{1}$ as their tangent space). Now $V, \Gamma$ and $\sigma=W\lrcorner \Omega$ all restrict to leaves of the foliation since $V$ and $\Gamma$ are tangent to the leaves and $W$ is in the kernel of $\sigma$. Denote by $V_{L}, \Gamma_{L}$ and $\sigma_{L}$ their restrictions to some leaf $L$ of $\mathscr{F}$. Then $\sigma_{L}$ is now Frobenius integrable on $L$ and thus

$$
\frac{\sigma_{L}}{\left.V_{L}\right\lrcorner \sigma_{L}}
$$

is closed on $L$, but this is just the restriction of $\omega^{2}$ to $L$. The decomposition of the exterior derivative can be written as $d=d_{1}+\hat{d}_{1}$ with the restriction of $d_{1}$ 
to $L$ being the exterior derivative on $L$ and $\hat{d}_{1}$ being characterised on 0 -forms $f \in \Lambda^{0} U$ by $\hat{d}_{1} f=W(f) \omega^{1}$. Now the converse of the Poincaré Lemma holds on each leaf $L$ of $\mathscr{F}$ so that $d_{1} \omega^{2}=0$ implies that $\omega^{2}=d_{1} \gamma_{L}^{2}$ for some $\gamma_{L}^{2}$ on each leaf, and by smoothness we must have $\gamma_{L}^{2}$ as the restriction of some function defined locally on the base manifold. Thus $\omega^{2}=d_{1} \gamma^{2}=d \gamma^{2}-\hat{d}_{1} \gamma^{2}=$ $d \gamma^{2}-W\left(\gamma^{2}\right) d \gamma^{1}$.

This result could alternatively have been proved using the divisibility of a Frobenius integrable form by one of its characterising 1 -forms. It is easy to see that locally we have $\omega^{1}=d \gamma^{1}$ and $\omega^{2}=d \gamma^{2}-W\left(\gamma^{2}\right) d \gamma^{1}$ with $W\left(\gamma^{1}\right)=$ $V\left(\gamma^{2}\right)=1$ and $V\left(\gamma^{1}\right)=0$.

In particular, these results hold for the prolongations of point symmetries $V$ and $W$ of (2-1) from $J^{0}(\mathbf{R}, \mathbf{R})$ to $U$ which satisfy $[V, W]=\alpha V$ for some $\alpha \in \Lambda^{0} J^{0}(\mathbf{R}, \mathbf{R})$. (A point vectorfield is one defined on the base, $J^{0}(\mathbf{R}, \mathbf{R})$; it can be prolonged to a vectorfield on a higher order jet bundle, see Olver [1] for the prolongation formulae.) That is, a solvable algebra of point symmetries satisfying the dimensional requirement provide a complete reduction to quadratures. We remind the reader that an $n$-dimensional (Lie) algebra is solvable if it has a chain of $n-1$ subalgebras, each of 1 dimension less than the previous, such that each element of the algebra is a symmetry of the next lowerdimensional one (in the usual terminology, each subalgebra is an ideal of the next higher-dimensional one).

A good example is provided by Olver [1, p. 147] with the second order equation

$$
x^{2} y^{\prime \prime}+\left(y^{\prime}\right)^{2} x=y^{\prime} y
$$

which we represent on $U=\{(x, y, p): x \neq 0\} \subset J^{1}(\mathbf{R}, \mathbf{R})$. Putting $f=$ $p(y-p x) / x^{2}$ then we have $\Gamma=\frac{\partial}{\partial x}+p \frac{\partial}{\partial y}+f \frac{\partial}{\partial p}, \Omega=(d y-p d x) \wedge(d p-f d x)$. This has the scaling point symmetry $X=x \frac{\partial}{\partial x}+y \frac{\partial}{\partial y}$ on the base manifold $\pi(U)=\left\{(x, y) \in J^{0}(\mathbf{R}, \mathbf{R}): x \neq 0\right\}$, whose prolongation to $U$ takes the same form and which we also denote by $X$.

Proposition (3-1) suggests we can relax our search for a second symmetry of $\Gamma$ by looking instead for a symmetry of $\operatorname{span}(\{\Gamma, X\})$, which is an easier condition to satisfy. We have in particular $Z=p \frac{\partial}{\partial p}$ with the commutator relations given by

$$
[X, \Gamma]=-\Gamma, \quad[Z, \Gamma]=\frac{x p}{x p-y} \Gamma-\frac{p}{x p-y} X, \quad[X, Z]=0
$$

so that $\mathscr{L}_{Z} \operatorname{span}(\{\Gamma, X\})=\operatorname{span}(\{\Gamma, X\})$. Now $\{X, Z, \Gamma\}$ are not linearly independent on the whole of $U$ so we restrict ourselves to $U^{\prime}=\{(x, y, p) \in$ $\left.J^{1}(\mathbf{R}, \mathbf{R}): x \neq 0, p \neq 0, x p \neq y\right\}$ thus excluding the solutions $y=k_{1} x$ and $y=k_{2}$ for $k_{1}, k_{2} \in \mathbf{R}$ which satisfy (3-2) on all of $\mathbf{R}^{2}$. We then have the two 1 -forms

$$
\omega^{1}=\frac{X\lrcorner \Omega}{Z\lrcorner X\lrcorner \Omega}=-\frac{y}{x^{2}} d x+\frac{1}{x} d y+\frac{1}{p} d p
$$

and

$$
\omega^{2}=\frac{Z\lrcorner \Omega}{X\lrcorner Z\lrcorner \Omega}=\frac{d y-p d x}{y-p x}
$$


with $d \omega^{1}=0$ and $d \omega^{2}=0 \bmod \omega^{1}$. This yields $\omega^{1}=d \gamma^{1}$ and $\omega^{2}=$ $d \gamma^{2}-Z\left(\gamma^{2}\right) d \gamma^{1}$ with

$$
\gamma^{1}=\frac{y}{x}+\log |p|
$$

and

$$
\gamma^{2}=\log |x|+\int_{y_{0} / x_{0}}^{y / x} \frac{d z}{A e^{z}+z} \text { where } A= \pm e^{-\gamma^{1}} .
$$

This integral is defined on each connected component of $U^{\prime}$, but there is no guarantee that it can be smoothly extended to all of $U$. Note that $Z-Z\left(\gamma^{2}\right) X$ is a symmetry but incredibly hard to find compared to $Z$. Actually, the given expressions for $\gamma^{1}$ and $\gamma^{2}$ are representatives of their equivalence classes: $\gamma^{1}$ is determined by $X$ and $Z$ up to the addition of an arbitrary constant, and $\gamma^{2}$ is determined up to addition of an arbitrary smooth function of $\gamma^{1}$. The general solution is given implicitly by treating $\gamma^{1}$ and $\gamma^{2}$ as constants (of integration); eliminating $p$ between them to give $y$ as a function of $x$ then gives the explicit solutions.

It is worth seeing how the approach here relates to Olver's method. To make the pass to the quotient transparent we introduce new canonical coordinate functions for $U^{\prime}$, namely

$$
u=\frac{y}{x}, \quad v=\log x, \quad \text { and } \quad w=\frac{d v}{d u}=\frac{x}{x p-y} .
$$

The coordinate representations of $\Gamma, X$ and $Z$ then become

$$
\begin{aligned}
& \Gamma=\frac{e^{-v}}{w}\left(\frac{\partial}{\partial u}+w \frac{\partial}{\partial v}+\left(w^{2} u+w^{2}+w\right) \frac{\partial}{\partial w}\right), \\
& X=\frac{\partial}{\partial v}, \quad Z=-w(1+u w) \frac{\partial}{\partial w} .
\end{aligned}
$$

Denoting the quotient manifold of $U^{\prime}$ by the group action of $X$ as $Q$, it has the global coordinates $(u, w)$. The condition for a vectorfield $V$ on $U^{\prime}$ to pass to $Q$ is that $\mathscr{L}_{X} V$ be some multiple of $X$. $\Gamma$ does not pass to $Q$, however we can always find a multiple of $\Gamma$ which does, in this case, we use $\widetilde{\Gamma}=w e^{v} \Gamma . Z$ passes to the quotient, and so does $[Z, \widetilde{\Gamma}]=-w(1+u w) X$.

On the quotient manifold $Q, \widetilde{\Gamma}$ and $Z$ become

$$
\widehat{\Gamma}=\frac{\partial}{\partial u}+\left(w^{2} u+w^{2}+w\right) \frac{\partial}{\partial w}, \quad \widehat{Z}=-w(1+u w) \frac{\partial}{\partial w}
$$

respectively with $[\hat{Z}, \hat{\Gamma}]=0$. Olver passes to the quotient and then looks for a symmetry of the reduced differential equation $\widehat{\Gamma}$, this is the vectorfield $\hat{Z}$. A first integral follows by the first order integrating factor result, this integral is just $\gamma^{1}$ on $Q$. Once we have identified that $Z$ is the unique vectorfield projecting to $\widehat{Z}$ with no $X$ component we can do away with the technique of passing to the quotient manifold to look for a symmetry of $\widehat{\Gamma}$ by simply looking for symmetries of both $\Gamma$ and its symmetries together. This largely obviates the necessity for canonical coordinates.

One last comment about this example that will be useful later: our emphasis here is on the simple form $X\lrcorner \Omega$, we regard the Pfaffian equation " $X\lrcorner \Omega=0$ " 
as the reduced differential equation rather than $\widetilde{\Gamma}$. This is justifiable because in canonical coordinates this Pfaffian equation is

$$
X\lrcorner \Omega=\frac{e^{v}}{w^{3}}\left(\left(w^{2} u+w^{2}+w\right) d u-d w\right)=0
$$

and the multiple $(X\lrcorner \Omega) / \Omega\left(\frac{\partial}{\partial w}, X\right)$ passes to the quotient as

$$
\left(w^{2} u+w^{2}+w\right) d u-d w=0
$$

with kernel spanned by $\widehat{\Gamma}$. So we treat “ $X\lrcorner \Omega$ ” as the reduced differential equation even though it lives on $U^{\prime}$ with kernel $\operatorname{span}(\{\Gamma, X\})$.

\section{GenERALISATIONS}

In this section we give the reduction results without the connections with differential equations. By being as general as possible, we can formulate the method and then illustrate it in the next section with the applications to ordinary differential equations. We begin with some basics on our generic manifold $M$.

The kernel or characteristic space of a differential form $\Omega$ is the span of vectorfields which annihilate $\Omega, \operatorname{ker} \Omega=\{X \in \mathfrak{X}(M): X\lrcorner \Omega=0\}$. A differential $p$-form $\Omega$ on an $n$ dimensional manifold is simple or decomposable if it is the wedge product of $p 1$-forms, so $\Omega$ is locally simple if $\operatorname{ker} \Omega$ is everywhere $n-p$ dimensional. If $\Omega$ is locally simple and we have a basis for its kernel which can be extended to a basis for the module of vectorfields $\mathfrak{X}(M)$ then $\Omega$ is simple, but we can only be sure of doing this locally. A constraint 1-form $\sigma$ for $\Omega$ is a 1-form satisfying $\sigma \wedge \Omega=0$, which implies $X\lrcorner \sigma=0$ for all $X \in \operatorname{ker} \Omega$. A 1-form factor for a simple form is a constraint 1-form, so that a simple form is a wedge product of constraint 1 -forms. A distribution $\mathscr{D}$ is an assignment of a vector subspace of $T_{p} M$ to each $p \in M$, and when regarded as a vector subspace of $\mathfrak{X}(M)$, is in involution if $[X, Y] \in \mathscr{D}$ for each $X, Y \in \mathscr{D}$. By the Frobenius theorem, $\mathscr{D}$ then spans the tangent space to an integral submanifold, which has the same dimension as $\mathscr{D}$, and we then say $\mathscr{D}$ is Frobenius integrable provided this dimension is constant. A $p$-form $\Omega$ is Frobenius integrable if its kernel is Frobenius integrable and of maximal dimension everywhere. The rank of a form is the codimension of its kernel, so a $p$-form $\Omega$ is Frobenius integrable if its kernel is Frobenius integrable and of minimal rank everywhere. The Frobenius theorem then guarantees that locally $\Omega$ is some multiple of the wedge product of $p$ exact 1-forms $\Omega=\gamma^{0} d \gamma^{1} \wedge d \gamma^{2} \wedge \cdots \wedge d \gamma^{p}$. Thus Frobenius integrability implies local simplicity. $X$ is a symmetry of a distribution $\mathscr{D}$ if $\mathscr{L}_{X} \mathscr{D}=\mathscr{D}$, i.e. $\mathscr{L}_{X} Y \in \mathscr{D}$ for all $Y \in \mathscr{D} . X$ is a symmetry of a differential form $\Omega$ if $\mathscr{L}_{X} \Omega=\lambda \Omega$ for some function $\lambda$. A foliation of a manifold $M$ is a Frobenius integrable distribution $\mathscr{D}$ on $M$, the leaves of the foliation are the integral submanifolds of $\mathscr{D}$, and we say $\mathscr{D}$ foliates $M$.

The following propositions, some of which we present without proof, are useful for understanding the first and second order theory already presented. They are also necessary for the material in $\S \mathrm{V}$.

Proposition 4.1. $X$ is a symmetry of a locally simple form $\Omega$ if and only if $X$ is a symmetry of $\operatorname{ker} \Omega$.

Proof. We note that if $Y \in \operatorname{ker} \Omega$ and $\sigma$ is any constraint 1 -form for $\Omega$ then $\left.Y\lrcorner \mathscr{L}_{X} \sigma=-\left(\mathscr{L}_{X} Y\right)\right\lrcorner \sigma$. So $\mathscr{L}_{X} Y \in \operatorname{ker} \Omega$ for all $Y \in \operatorname{ker} \Omega$ implies $\mathscr{L}_{X} \sigma$ is a 
constraint 1-form for $\Omega$ (or zero). Clearly if 1 -form factors are invariant under $X$ then $\mathscr{L}_{X} \Omega=\lambda \Omega$ for some $\lambda$ and so $X$ is a symmetry of $\Omega$. Conversely if $\mathscr{L}_{X} \Omega=\lambda \Omega$ for some $\lambda$ then $\left.\left.0=\mathscr{L}_{X}(Y\lrcorner \Omega\right)=\left(\mathscr{L}_{X} Y\right)\right\lrcorner \Omega$ for $Y \in \operatorname{ker} \Omega$ implying $\mathscr{L}_{X} Y \in \operatorname{ker} \Omega$ and $X$ is a symmetry of $\operatorname{ker} \Omega$.

Proposition 4.2. If $X\lrcorner \Omega \neq 0$ everywhere on $M$ then $X\lrcorner \Omega$ is locally simple if and only if $\Omega$ is locally simple.

Proof. Since $\operatorname{ker}(X\lrcorner \Omega) \supseteq \operatorname{span}(\{X\} \cup \operatorname{ker} \Omega)$ then $X\lrcorner \Omega \neq 0$ ensures that the condition $\operatorname{ker}(X\lrcorner \Omega)$ has maximal dimension is equivalent to $\operatorname{ker} \Omega$ having maximal dimension.

Proposition 4.2 is trivial for the second order equations of $\S I I I$, but we do need it for systems of equations.

Proposition 4.3. If $\Omega$ is a p-form, and $X_{1}, \ldots, X_{k}$ are vectorfields linearly independent everywhere with $\left.X_{i}\right\lrcorner \Omega \neq 0$ everywhere and $k \leq p$ then $\left.\left.X_{1}\right\lrcorner X_{2}\right\lrcorner \ldots$ \lrcorner $\left.X_{k}\right\lrcorner \Omega$ is locally simple if and only if $\Omega$ is locally simple.

Proposition 4.4. If $\Omega$ is a locally simple p-form on an m-dimensional manifold $M$ and $X_{1}, \ldots, X_{k}$ are linearly independent vectorfields satisfying $\left.X_{i}\right\lrcorner \Omega \neq 0$ everywhere and $k \leq p$ then $\left.\left.\left.\left.X_{1}\right\lrcorner X_{2}\right\lrcorner \ldots\right\lrcorner X_{k}\right\lrcorner \Omega$ is Frobenius integrable if and only if $\operatorname{span}\left(\left\{X_{1}, \ldots, X_{k}\right\} \cup \operatorname{ker} \Omega\right)$ is Frobenius integrable.

Proposition 4.5. If $\Omega$ is Frobenius integrable and $X$ is a symmetry of $\Omega$ with $X\lrcorner \Omega \neq 0$ everywhere then $X\lrcorner \Omega$ is Frobenius integrable.

Proof. We have $X$ as a symmetry of $\operatorname{ker} \Omega$ and the Lie bracket is closed on $\operatorname{ker} \Omega$ so the Lie bracket is also closed on $\operatorname{span}(\{X\} \cup \operatorname{ker} \Omega)=\operatorname{ker}(X\lrcorner \Omega)$ which is then Frobenius integrable by definition.

Proposition 4.6. If $\Omega$ is Frobenius integrable and $\left\{X_{1}, \ldots, X_{k}\right\}$ is a basis for a $k$-dimensional algebra of linearly independent symmetries of $\Omega$ satisfying $\left.X_{i}\right\lrcorner \Omega \neq 0$ everywhere then $\left.\left.\left.\left.X_{1}\right\lrcorner X_{2}\right\lrcorner \ldots\right\lrcorner X_{k}\right\lrcorner \Omega$ is Frobenius integrable.

Proposition 4.6 is a generalisation of Corollary 3.2.

Proposition 4.7. Let $\Omega$ be a $k$-form on a manifold $M$, and $\operatorname{span}\left(\left\{X_{1}, \ldots, X_{k}\right\}\right)$ be a $k$-dimensional distribution on open $U \subseteq M$ satisfying $\left.X_{i}\right\lrcorner \Omega \neq 0$ everywhere on $U$. Further suppose that $\operatorname{span}\left(\left\{X_{j+1}, \ldots, X_{k}\right\} \cup \operatorname{ker} \Omega\right)$ is Frobenius integrable for some $j<k$ and that $X_{i}$ is a symmetry of $\operatorname{span}\left(\left\{X_{i+1}, \ldots, X_{k}\right\} \cup\right.$ $\operatorname{ker} \Omega)$ for $i=1, \ldots, j$. Put

$$
\begin{aligned}
\sigma^{1} & \left.\left.\left.\left.=X_{2}\right\lrcorner X_{3}\right\lrcorner \ldots\right\lrcorner X_{k}\right\lrcorner \Omega, \\
\sigma^{2} & \left.\left.\left.\left.=X_{1}\right\lrcorner X_{3}\right\lrcorner \ldots\right\lrcorner X_{k}\right\lrcorner \Omega, \\
& \vdots \\
\sigma^{k} & \left.\left.\left.\left.=X_{1}\right\lrcorner X_{2}\right\lrcorner \ldots\right\lrcorner X_{k-1}\right\lrcorner \Omega, \\
\omega^{i} & =\frac{\sigma^{i}}{\left.X_{i}\right\lrcorner \sigma^{i}} \quad \text { for } i=1, \ldots, k
\end{aligned}
$$

so that $\left\{\omega^{1}, \ldots, \omega^{k}\right\}$ is dual to $\left\{X_{1}, \ldots, X_{k}\right\}$. Then $d \omega^{1}=0 ; d \omega^{2}=0$ 
$\bmod \omega^{1} ; \ldots ; d \omega^{j}=0 \bmod \omega^{1}, \ldots, \omega^{j-1}$ so that locally

$$
\begin{aligned}
& \omega^{1}=d \gamma^{1}, \\
& \omega^{2}=d \gamma^{2}-X_{1}\left(\gamma^{2}\right) d \gamma^{1}, \\
& \omega^{3}=d \gamma^{3}-X_{2}\left(\gamma^{3}\right) d \gamma^{2}-\left(X_{1}\left(\gamma^{3}\right)-X_{2}\left(\gamma^{3}\right) X_{1}\left(\gamma^{2}\right)\right) d \gamma^{1}, \\
& \quad \vdots \\
& \omega^{j}=d \gamma^{j} \bmod d \gamma^{1}, \ldots, d \gamma^{j-1}
\end{aligned}
$$

for some $\gamma^{1}, \ldots, \gamma^{j} \in \Lambda^{0} T^{*} U$. Also the system $\left\{\omega^{j+1}, \ldots, \omega^{k}\right\}$ is Frobenius integrable modulo $d \gamma^{1}, \ldots, d \gamma^{j}$ and locally $\Omega=\gamma^{0} d \gamma^{1} \wedge d \gamma^{2} \wedge \cdots \wedge d \gamma^{j} \wedge$ $\omega^{j+1} \wedge \cdots \wedge \omega^{k}$ for some $\gamma^{0} \in \Lambda^{0} T^{*} U$. Each $\gamma^{i}$ is uniquely defined up to the addition of an arbitrary function of $\gamma^{1}, \ldots, \gamma^{i-1}$.

Proof. $\operatorname{span}\left(\left\{X_{j+1}, \ldots, X_{k}\right\} \cup \operatorname{ker} \Omega\right)$ is Frobenius integrable with symmetry $X_{j}$, so $\operatorname{span}\left(\left\{X_{j}, \ldots, X_{k}\right\} \cup \operatorname{ker} \Omega\right)$ is Frobenius integrable and recursively $\operatorname{span}\left(\left\{X_{i}, \ldots, X_{k}\right\} \cup \operatorname{ker} \Omega\right)$ is Frobenius integrable for $i=1, \ldots, j$. Then $\sigma^{1}$ is Frobenius integrable and has symmetry $X_{1}$ so $\omega^{1}$ is closed, and locally $\omega^{1}=d \gamma^{1}$ for some $\gamma^{1} \in \Lambda^{0} T^{*} U$. Consider the foliation $\mathscr{F}^{1}$ of $U$ by $\operatorname{ker} \omega^{1}$. Restricting $\sigma^{2}$ to the leaves $L_{1}$ of $\mathscr{F}^{1}$ (which are spanned by $X_{2}, \ldots, X_{k}$ and $\operatorname{ker} \Omega)$ then $\sigma^{2}$ has Frobenius integrable kernel $\operatorname{span}\left(\left\{X_{3}, \ldots, X_{k}\right\} \cup \operatorname{ker} \Omega\right)$ on $L_{1}$ and symmetry $X_{2}$ so $\omega^{2}$ is closed on each leaf of $\mathscr{F} 1$. We can decompose the exterior derivative $d$ on $U$ into $d=d_{1}+\hat{d}_{1}$ where $d_{1}$ is the foliated exterior derivative on leaves of $\mathscr{F}^{1}$, and where $\hat{d}_{1}$ is characterised on 0 -forms by $\hat{d}_{1} f=X_{1}(f) \omega^{1}$. So we have $d_{1} \omega^{2}=0$ and $d \omega^{2}=0 \bmod \omega^{1}$. Now the converse of Poincare's Lemma holds on leaves of $\mathscr{F}_{1}$ so $d_{1} \omega^{2}=0$ implies locally $\omega^{2}=d_{1} \gamma^{2}$ and using the decomposition $d=d_{1}+\hat{d}_{1}$ we have $\omega^{2}=$ $d \gamma^{2}-\hat{d}_{1} \gamma^{2}=d \gamma^{2}-X^{1}\left(\gamma^{2}\right) d \gamma^{1}$. Likewise, foliating $U$ by $\operatorname{ker} \omega^{1} \wedge \omega^{2}$ we have the decomposition $d=d_{2}+\hat{d}_{2}$ where $d_{2}$ is the foliated exterior derivative on leaves of $\mathscr{F}^{2}$, and where $\hat{d}_{2}$ is characterised on 0 -forms by $\hat{d}_{2} f=X_{1}(f) \omega^{1}+$ $X^{2}(f) \omega^{2}$. Thus we have $d_{2} \omega^{3}=0$ implying $d \omega^{3}=0 \bmod \omega^{1}, \omega^{2}$ and locally $\omega^{3}=d \gamma^{3}-X^{2}\left(\gamma^{3}\right) \omega^{2}-X^{1}\left(\gamma^{3}\right) \omega^{1}$. Clearly the process is inductive, and then the proof is complete.

Proposition 4.7 is the generalisation of Corollary 3.4. This result would usually be used with a full set of symmetries, in which case it says that a basis for $\mathfrak{X}(U)$ consisting of $\Gamma$ and symmetries, produces a dual basis containing the Cartan invariant 1-forms, or basic forms as they are sometimes called. These forms are precisely the pullbacks by the natural projection of 1-forms on the quotient manifold of $M$ by the group action of $\Gamma$.

\section{ORDINARY DIFFERENTIAL EQUATIONS AGAIN}

Here we present the results of the last section as they apply to ordinary differential equations. The generalisation to systems of equations and to partial differential equations is indicated, although the focus is on ordinary differential equations. We begin by setting up the notation for an $n$th order ordinary differential equation.

Definition. Let $x, y^{a}, y^{a(1)}, \ldots, y^{a(n-1)}$ for $a=1, \ldots, m$ be natural coordinates on the $(n-1)$ th jet bundle $J^{n-1}\left(\mathbf{R}, \mathbf{R}^{m}\right)$. Put $\sigma_{1}^{a}=d y^{a}-y^{a(1)} d x$, 
$\sigma_{j}^{a}=d y^{a(j-1)}-y^{a(j)} d x$. Then $\left\{\sigma_{1}^{a}, \ldots, \sigma_{n-1}^{a}\right\}_{a=1}^{m}$ is the natural basis for the contact forms on $J^{n-1}\left(\mathbf{R}, \mathbf{R}^{m}\right)$, which are the forms on $J^{n-1}\left(\mathbf{R}, \mathbf{R}^{m}\right)$ which are zero on curves or surfaces lifted from $J^{0}\left(\mathbf{R}, \mathbf{R}^{m}\right)$. They give meaning to the derivative coordinates, such as $y^{a(1)}:=\left(y^{a}\right)^{\prime}$ as being the derivative of $y^{a}$. Let $f^{a} \in \Lambda^{0} J^{n-1}\left(\mathbf{R}, \mathbf{R}^{m}\right)$ then put $\sigma_{n}^{a}=d y^{a(n-1)}-f^{a} d x$. Then the $\sigma_{n}^{a}$ 's are the natural force forms for the system of $m n$th order differential equations

$$
\frac{d^{n} y^{a}}{d x^{n}}=f^{a}\left(x, y^{a}, \ldots, y^{a(n-1)}\right)
$$

on $J^{n-1}\left(\mathbf{R}, \mathbf{R}^{m}\right)$. Put

$$
\Omega=\bigwedge_{a, j} \sigma_{j}^{a}
$$

meaning the $m n$-fold wedge product, then $\Omega$ is the natural characterising form for $(5-1)$,

$$
\Gamma=\frac{\partial}{\partial x}+y^{a(1)} \frac{\partial}{\partial y^{a}}+y^{a(2)} \frac{\partial}{\partial y^{a(1)}}+\cdots+f^{a} \frac{\partial}{\partial y^{a(n-1)}} .
$$

Then $\Gamma$ is an $n$th order differential equation field, it is the characteristic vectorfield of (5-1), tangent to the lifted solution curves and represents differentiation along the solution curves. It is the vectorfield in the kernel of $\Omega$ normalised by $\Gamma\lrcorner d x=1$. We can rewrite (5-1) as a Pfaffian equation " $\Omega=0$ ".

We remark in passing that any volume form $\Theta$ on $J^{n-1}\left(\mathbf{R}, \mathbf{R}^{m}\right)$ provides a characterising form $\Gamma\lrcorner \Theta$ for (5-1) as this is just some nonzero multiple of $\Omega$. Indeed, the generalisation to partial differential equations involves the interior product of such a volume form with all of the characteristic vectorfields of the equation.

A symmetry of (5-1) is any map of $J^{0}\left(\mathbf{R}, \mathbf{R}^{m}\right)$ into itself which permutes the solution curves of (5-1). In particular, a one-parameter group action on $J^{n-1}\left(\mathbf{R}, \mathbf{R}^{m}\right)$, generated by a vectorfield $X$, with the property $\mathscr{L}_{X} \Gamma=\lambda \Gamma$ will permute the integral curves of $\Gamma$ and hence, by projection, the solution curves on $J^{0}\left(\mathbf{R}, \mathbf{R}^{m}\right)$. Such a vectorfield is also called a symmetry, and this is the standard idea that we use. The group action is said to be projectable if the action on the base is that of a one-parameter group and $X$ is also said to be projectable; however in general this will not occur. Projectable symmetries are known as point symmetries and we usually begin with the action on the base and prolong it to $J^{n-1}\left(\mathbf{R}, \mathbf{R}^{m}\right)$. The prolongation formulae require that the prolonged action preserve the contact structure. Proposition 4.1 guarantees that a symmetry $X$ satisfies $\mathscr{L}_{X} \Omega=\bar{\lambda} \Omega$ and vice versa.

It is appropriate to now discuss the traditional notion of symmetry of a differential equation. Classically, an $n$th order (partial) differential equation for $r$ unknowns in $s$ variables is a function $F$ (or system of functions) on the $n$th jet space $J^{n}\left(\mathbf{R}^{r}, \mathbf{R}^{s}\right)$, the solutions being the projection to $J^{0}\left(\mathbf{R}^{r}, \mathbf{R}^{s}\right)$ of $r$-dimensional submanifolds of $J^{n}\left(\mathbf{R}^{r}, \mathbf{R}^{s}\right)$ in the zero hypersurface of $F$. A symmetry of this differential equation is a transformation on $J^{0}\left(\mathbf{R}^{r}, \mathbf{R}^{s}\right)$ which maps solution surfaces to other solution surfaces. A vectorfield $V$ on $J^{n}\left(\mathbf{R}^{r}, \mathbf{R}^{s}\right)$ is then a symmetry of the differential equation " $F=0$ " if and only if it is tangent to the zero set of $F$ on $J^{n}\left(\mathbf{R}^{r}, \mathbf{R}^{s}\right)$, which means the group 
action which $V$ generates will map this zero set into itself; and this is equivalent to $V(F)=0$ whenever $F=0$. An explicit differential equation has all $n$th order derivatives specified explicitly in terms of at most $(n-1)$ th order derivatives, such a differential equation has the advantage of a nice geometrical setting on the $(n-1)$ th jet space $J^{n-1}\left(\mathbf{R}^{r}, \mathbf{R}^{s}\right)$. Here, we have the $r$ total derivative operators of differentiation on solution surfaces existing as vectorfields, these form a Frobenius integrable distribution (provided the equations are consistent) tangent to solution surfaces. The differential equation is then specified by the characteristic vectorfields $\Gamma^{1}, \ldots, \Gamma^{r}$ or by a single Frobenius integrable differential form $\Omega$ which is zero on the solution surfaces. A general solution to the differential equation is then provided by finding all the first integrals. It can then be shown that symmetries of the differential equation are just the symmetries of $\Omega$.

The following results are essentially corollaries of some of the propositions in $\S I V$ in the case where $\Omega$ corresponds to an $n$th order equation field $\Gamma$.

Proposition 5.1. Let $X_{1}, \ldots, X_{m n}$ form an abelian algebra of symmetries of (5-1) on $U \subseteq J^{n-1}\left(\mathbf{R}, \mathbf{R}^{m}\right)$, which together with $\Gamma$ form a basis for $\mathfrak{X}(U)$. Then take $\left\{\omega^{0}, \omega^{1}, \ldots, \omega^{m n}\right\}$ to be the basis for $\wedge^{1} U$ dual to $\left\{\Gamma, X_{1}, \ldots, X_{m n}\right\}$. Then we have $d \omega^{i}=0$ for $i=1, \ldots, m n$ and locally $\omega^{i}=d \gamma^{i}$ such that the $\gamma^{i}$ 's form a complete set of functionally independent invariants for (5-1) and thus constitute a general solution.

Corollary 5.2. If (5-1) has an mn-dimensional abelian algebra of symmetries then the differential equation is reduced to quadratures.

Proposition 5.3. Let $\left\{X_{1}, \ldots, X_{m n}\right\}$ form an ordered basis for a solvable algebra of symmetries of (5-1) on $U \subseteq J^{n-1}\left(\mathbf{R}, \mathbf{R}^{m}\right)$, which together with $\Gamma$ form a basis for $\mathfrak{X}(U)$. Take $\left\{\omega^{0}, \omega^{1}, \ldots, \omega^{m n}\right\}$ to be the basis for $\wedge^{1} U$ dual to $\left\{\Gamma, X_{1}, \ldots, X_{m n}\right\}$. Then $d \omega^{1}=0 ; d \omega^{2}=0 \bmod \omega^{1} ; \ldots ; d \omega^{m n}=0$ $\bmod \omega^{1}, \ldots, \omega^{m n-1}$ so that locally $\omega^{1}=d \gamma^{1}, \omega^{2}=d \omega^{2}-X^{1}\left(\gamma^{2}\right) d \gamma^{1} ; \ldots$; $\omega^{m n}=d \gamma^{m n} \bmod \gamma^{1}, \ldots, \gamma^{m n-1}$ for some functions $\gamma^{1}, \ldots, \gamma^{m n} \in \Lambda^{0} U$. Locally $\Omega=\gamma^{0} d \gamma^{1} \wedge d \gamma^{2} \wedge \cdots \wedge d \gamma^{m n}$ for some function $\gamma^{0} \in \wedge^{0} U$. Each $\gamma^{i}$ is uniquely defined up to the addition of an arbitrary function of $\gamma^{1}, \ldots, \gamma^{i-1}$, and these constitute a general solution to (5-1).

Corollary 5.4. If (5-1) has an mn-dimensional solvable algebra of linearly independent symmetries then the differential equation is reduced to quadratures.

This corollary is a generalisation of the classical result that an $n$th order system of $m$ differential equations, with an $m n$-dimensional solvable algebra of point symmetries whose $n$th prolongation has $m n$-dimensional orbits, is reduced to quadratures. Clearly the condition on the dimension of the orbits of the prolonged algebra is equivalent to requiring that our $m n$ prolonged symmetries are everywhere linearly independent, or that they can be used to form a basis for the module of vectorfields.

The next result to give is the most general result for the reduction of a differential equation, not necessarily using symmetries.

Proposition 5.5. If $\operatorname{span}\left(\left\{X_{1}, \ldots, X_{m n}\right\}\right)$ is a mn-dimensional distribution on an open $U \subseteq M$ satisfying $\left.X_{i}\right\lrcorner \Omega \neq 0$ everywhere on $U$ and

$$
\operatorname{span}\left(\left\{X_{j+1}, \ldots, X_{m n}, \Gamma\right\}\right)
$$


is Frobenius integrable for some $j<m n$ with $X_{i}$ a symmetry of

$$
\operatorname{span}\left(\left\{X_{i+1}, \ldots, X_{m n}, \Gamma\right\}\right)
$$

for $i=1, \ldots, j$, then put

$$
\begin{aligned}
\sigma^{1} & \left.\left.\left.\left.=X_{2}\right\lrcorner X_{3}\right\lrcorner \ldots\right\lrcorner X_{m n}\right\lrcorner \Omega, \\
\sigma^{2} & \left.\left.\left.\left.=X_{1}\right\lrcorner X_{3}\right\lrcorner \ldots\right\lrcorner X_{m n}\right\lrcorner \Omega, \\
& \vdots \\
\sigma^{k} & \left.\left.\left.\left.=X_{1}\right\lrcorner X_{2}\right\lrcorner \ldots\right\lrcorner X_{m n-1}\right\lrcorner \Omega, \\
\omega^{i} & =\frac{\sigma^{i}}{\left.X_{i}\right\lrcorner \sigma^{i}} \quad \text { for } i=1, \ldots, m n
\end{aligned}
$$

so that $\left\{\omega^{1}, \ldots, \omega^{m n}\right\}$ is dual to $\left\{X_{1}, \ldots, X_{m n}\right\}$.

Then $d \omega^{1}=0, d \omega^{2}=0 \bmod \omega^{1} ; \ldots ; d \omega^{j}=0 \bmod \omega^{1}, \ldots, \omega^{j-1}$ so that locally

$$
\begin{aligned}
& \omega^{1}=d \gamma^{1} \\
& \omega^{2}=d \gamma^{2}-X_{1}\left(\gamma^{2}\right) d \gamma^{1}, \\
& \omega^{3}=d \gamma^{3}-X_{2}\left(\gamma^{3}\right) d \gamma^{2}-\left(X_{1}\left(\gamma^{3}\right)-X_{2}\left(\gamma^{3}\right) X_{1}\left(\gamma^{2}\right)\right) d \gamma^{1}, \\
& \quad \vdots \\
& \omega^{j}=d \gamma^{j} \bmod d \gamma^{1}, \ldots, d \gamma^{j-1}
\end{aligned}
$$

for some $\gamma^{1}, \ldots, \gamma^{j} \in \bigwedge^{0} T^{*} U$, which are then first integrals of (5-1). Each $\gamma^{i}$ is uniquely defined up to the addition of an arbitrary function of $\gamma^{1}, \ldots, \gamma^{i-1}$.

So far we have only considered the situation where we have no more symmetries than needed to perform the reduction, but if we have any extra symmetries these can be used to find first integrals of (5-1) without integration.

Proposition 5.6. Suppose we have integrable distribution $\mathscr{D}$ with $\Gamma \in \mathscr{D}$, and two symmetries $X$ and $Y$ of $\mathscr{D}$ which themselves are not in $\mathscr{D}$ which we can write as $Y=\alpha X+\beta Z$ for some $Z \in \mathscr{D}$. Then $\alpha$ is a (possibly trivial) first integral of (5-1).

Proof. We need only note that by assumption, $[Y, \Gamma]=-\Gamma(\alpha) X+\bar{Z}$, for some $\bar{Z} \in \mathscr{D}$, must also be in $\mathscr{D}$ so $\Gamma(\alpha)=0$.

The obvious corollary of this is when we have an over supply of point symmetries, or even when the linear independence condition fails.

Corollary 5.7. Suppose we have independent symmetries $X_{1}, \ldots, X_{j}$ of (5-1), and an additional symmetry $Y$ given by $Y=\alpha^{a} X_{a}+\beta \Gamma$. Then the $\alpha^{a}$ 's are (possibly trivial) first integrals of (5-1).

Proof. As above, noting that $[Y, \Gamma]=-\Gamma\left(\alpha^{a}\right) X_{a}+\varepsilon \Gamma$ must be a multiple of $\Gamma$ implies $\Gamma\left(\alpha^{a}\right)=0$.

Actually resolving the vectorfield $Y$ into components with respect to $X$ and $\Gamma$ might not be straightforward, however with the use of $\Omega$ we can find these components easily when we have an overabundance of symmetries. If we have 
vectorfields $\left\{X_{1}, \ldots, X_{m n}\right\}$ satisfying the hypothesis of Proposition 5.5 with corresponding first integral $\gamma^{1}$ and $\left\{Y, X_{2}, \ldots, X_{m n}\right\}$ also satisfying the hypothesis then we have $Y=\alpha X_{1}+\beta Z$ for some $Z \in \operatorname{span}\left(\left\{X_{2}, \ldots, X_{m n}, \Gamma\right\}\right)$. Then, by Proposition 5.6, $\alpha$ is a first integral of $(5-1)$. It is simple to check that $\alpha$ is the ratio

$$
Y\left(\gamma^{1}\right)=\frac{\left.\left.\left.\left.Y\lrcorner X_{2}\right\lrcorner X_{3}\right\lrcorner \ldots\right\lrcorner X_{m n}\right\lrcorner \Omega}{\left.\left.\left.\left.\left.X_{1}\right\lrcorner X_{2}\right\lrcorner X_{3}\right\lrcorner \ldots\right\lrcorner X_{m n}\right\lrcorner \Omega},
$$

the important thing here is that both these terms are integrating factors for the Frobenius integrable form $\sigma^{1}$ of Proposition 5.5 and thus we have a generalisation of the old result that the ratio of any two integrating factors for a first order differential equation gives a first integral of that equation. Cartan [4] gives a variety of similar ways of producing first integrals.

As an example of the results in this section, we look at one of the famous dynamical systems with symmetry, the Kepler problem. The calculations given are rather abridged for the sake of brevity, and we refer the reader to [7] for full details.

For simplicity we consider the planar problem in polar coordinates $(r, \theta)$. The pair of second order equations is

$$
\ddot{r}=r \dot{\theta}^{2}-\frac{\mu}{r^{2}}, \quad \ddot{\theta}=-\frac{2 \dot{r} \dot{\theta}}{r}
$$

with the second order equation field

$$
\Gamma=\frac{\partial}{\partial t}+\dot{r} \frac{\partial}{\partial r}+\dot{\theta} \frac{\partial}{\partial \theta}+\left(r \dot{\theta}^{2}-\frac{\mu}{r^{2}}\right) \frac{\partial}{\partial \dot{r}}-\frac{2 \dot{r} \dot{\theta}}{r} \frac{\partial}{\partial \dot{\theta}}
$$

and the associated locally simple 4 -form is

$$
\Omega=(d r-\dot{r} d t) \wedge(d \theta-\dot{\theta} d t) \wedge\left(d \dot{r}-\left(r \dot{\theta}^{2}-\frac{\mu}{r^{2}}\right) d \dot{\theta}\right) \wedge\left(d \dot{\theta}-\frac{2 \dot{r} \dot{\theta}}{r} d t\right) .
$$

The problem has exactly three point symmetries, namely,

$$
X_{1}=\frac{\partial}{\partial t}, \quad X_{2}=\frac{\partial}{\partial \theta}, \quad \text { and } \quad X_{3}=t \frac{\partial}{\partial t}+\frac{2}{3} r \frac{\partial}{\partial r}
$$

which form a solvable (but not nilpotent) algebra with commutators

$$
\left[X_{1}, X_{2}\right]=0, \quad\left[X_{2}, X_{3}\right]=0 \text { and }\left[X_{1}, X_{3}\right]=X_{1} .
$$

In addition, the field

$$
Z=\frac{1}{r^{4} \dot{\theta}^{2} \dot{r}} \frac{\partial}{\partial \dot{r}}
$$

is a symmetry of the integrable distribution $\mathscr{D}=\operatorname{span}\left(\left\{\Gamma, X_{1}, X_{2}, X_{3}^{(1)}\right\}\right)$ where $X_{3}^{(1)}$ is the first prolongation of $X_{3}$. On using the reduction of order algorithm of Proposition 5.5 we obtain the four 1 -forms

$$
\begin{array}{ll}
\omega^{1}=\frac{\left.\left.Z\lrcorner X_{2}\right\lrcorner X_{1}\right\lrcorner \Omega}{\Omega\left(X_{1}, X_{2}, Z, X_{3}^{(1)}\right)}, & \omega^{2}=\frac{\left.\left.\left.X_{3}^{(1)}\right\lrcorner X_{2}\right\lrcorner X_{1}\right\lrcorner \Omega}{\Omega\left(X_{1}, X_{2}, X_{3}^{(1)}, Z\right)}, \\
\omega^{3}=\frac{\left.\left.Z\lrcorner X_{3}^{(1)}\right\lrcorner X_{1}\right\lrcorner \Omega}{\Omega\left(X_{1}, X_{3}^{(1)}, Z, X_{2}\right)}, & \omega^{4}=\frac{\left.\left.Z\lrcorner X_{3}^{(1)}\right\lrcorner X_{2}\right\lrcorner \Omega}{\Omega\left(X_{2}, X_{3}^{(1)}, Z, X_{1}\right)} .
\end{array}
$$


$\omega^{1}$ and $\omega^{2}$ are closed and integrate locally to $\gamma^{1}=3 \log (h)$ and $\gamma^{2}=h^{2} E$ where $h=r^{2} \dot{\theta}$ and $E=\frac{1}{2}\left(\dot{r}^{2}+h^{2} / r^{2}\right)-\mu / r$ are the first integrals of angular momentum and energy respectively. $\omega^{3}$ and $\omega^{4}$ are closed modulo $d \gamma^{1}$ and $d \gamma^{2}$ and can be locally integrated to first integrals $\gamma^{3}$ and $\gamma^{4}$ representing initial values of $\theta$ and $t$ respectively. We can then use the local basis $\left\{d t, d \gamma^{1}, d \gamma^{2}, d \gamma^{3}, d \gamma^{4}\right\}$ to compute a dual basis $\left\{\Gamma, W_{1}, W_{2}, X_{2}, X_{1}\right\}$ where $W_{1}$ and $W_{2}$ are nonpoint symmetries of $\Gamma$ given by $W_{1}=Z-Z\left(\gamma^{4}\right) X_{1}-$ $Z\left(\gamma^{3}\right) X_{2}$ and $W_{2}=X_{3}^{(1)}-X_{3}^{(1)}\left(\gamma^{4}\right) X_{1}$. In this way we have a canonical reduction of order for the Kepler problem, the integrals $\gamma^{1}$ and $\gamma^{2}$ relating to the intrinsic geometry of the conic sections, and the integrals $\gamma^{3}$ and $\gamma^{4}$ relating to initial spatial and temporal conditions.

\section{CANONICAL COORDINATES}

In this section we make the connection with the traditional canonical coordinate method reduction of order of an ordinary differential equation using a point symmetry. We give a generalisation of the method for arbitrary symmetries which although follows easily from the point symmetry method, we believe it to be new. This provides a practical use for such symmetries in general; they usually appear only in the limited context of Noether's theorem. In the classical approach, we consider an implicit $n$th order equation on the $n$th jet bundle $J^{n}(\mathbf{R}, \mathbf{R})$, assumed totally nondegenerate, as

$$
F\left(x, y, y^{\prime}, \ldots, y^{(n)}\right)=0
$$

A solution of this equation is any subset of the zero set of $F: U_{0}=\{p \in$ $\left.J^{n}(\mathbf{R}, \mathbf{R}): F(p)=0\right\}$ on which the $n$th order contact structure is zero: by dimension the solutions must be curves.

Suppose we have a point symmetry $V$ of (6-1), that is a vectorfield $V$ on $J^{0}(\mathbf{R}, \mathbf{R})$ whose prolongation $V^{(n)}$ to $J^{n}(\mathbf{R}, \mathbf{R})$ is a symmetry of (6-1). The usual technique is to find the invariants of $V$ and $V^{(1)}$, and use these to construct a full set of invariants of $V^{(n)}$. We say $\zeta$ is a $j$ th order invariant of $V$ if $\zeta \in \Lambda^{0} J^{j}(\mathbf{R}, \mathbf{R})$ and $V^{(j)}(\zeta)=0$. Clearly a $j$ th order invariant is also a $(j+1)$ th order invariant (as usual we omit reference to the projection which is necessary when lifting an object from one jet space to a higher order one in the natural manner). The reduction is then performed by re-expressing $F$ in terms of the invariants of $V$, and this becomes the reduced differential equation.

Defining the total derivative operator

$$
D_{x}=\frac{\partial}{\partial x}+y^{\prime} \frac{\partial}{\partial y}+y^{\prime \prime} \frac{\partial}{\partial y^{\prime}}+\cdots+y^{(n+1)} \frac{\partial}{\partial y^{(n)}}+\ldots,
$$

it is well known that if $\xi$ and $\zeta$ are $j$ th order invariants of a symmetry $V$ then $D_{x}(\zeta) / D_{x}(\xi)$ is a $(j+1)$ th order invariant. Following Lie, we find a zeroth order invariant $u$ and a first order invariant $z$ for $V$. We define $z^{(0)}=z$ and $z^{(j)}=D_{x}\left(z^{(j-1)}\right) / D_{x}(u)$ for $j=1, \ldots, n$ so that we have the interpretation $z^{(j)}=d^{n} z / d u^{j}$. The classical result is that when we re-express $F$ in terms of $u, z, z^{\prime}, \ldots, z^{(n-1)},(6-1)$ becomes an $(n-1)$ th order equation for $z(u)$. Once we have solved this equation, the solution to (6-1) is given by integrating a related first order equation found by inverting the transformation to the invariants of $V$. 
In keeping with the theme of the paper, we choose to work with differential forms on the lower order jet space, so we assume that the differential equation can be written explicitly in the form $F\left(x, y, \ldots, y^{(n)}\right)=y^{(n)}-$ $f\left(x, y, \ldots, y^{(n-1)}\right)=0$ so that we are back in the scenario of $(5-1)$ with $m=1$. We make the identification by using the map $\tau: U_{0} \rightarrow J^{n-1}(\mathbf{R}, \mathbf{R})$ with $\tau\left(y^{(n)}\right)=f\left(x, y, \ldots, y^{(n-1)}\right)$.

To use canonical coordinates for the symmetry $V$ on $J^{n-1}(\mathbf{R}, \mathbf{R})$ we will express the Pfaffian equations for (5-1) in terms of the $n$th order invariants for $V$. The invariants of Proposition (6-1) can be defined by using $\Gamma$ instead of $D_{x}$, this will give the same invariants except that the $z^{(n-1)}$ defined using $\Gamma$ will be the pushforward from $J^{n}(\mathbf{R}, \mathbf{R})$ by the map $\tau$ of the $z^{(n-1)}$ defined using $D_{x}$. Denote this by $\hat{f}=\Gamma\left(z^{(n-2)}\right) / \Gamma(u) . \hat{f}$ is still an invariant of $V$, but it is functionally dependent on $u, z, z^{(1)}, \ldots, z^{(n-2)}$ and so we might write $\hat{f}=\hat{f}\left(u, z, z^{(1)}, \ldots, z^{(n-2)}\right)$.

Proposition 6.1. Suppose $u$ and $z$ are functionally independent zeroth order and first order invariants respectively of the point symmetry $V$, that is $V(u)=$ $V^{(1)}(z)=0$, and assume that $\Gamma(z)$ and $\Gamma(u)$ are nowhere zero. Put $z^{(0)}=z$ and $z^{(j)}=\Gamma\left(z^{(j-1)}\right) / \Gamma(u)$ for $j=1, \ldots, n-2$. Then $u, z, z^{(1)}, \ldots, z^{(n-2)}$ provide a complete set of functionally independent $(n-1)$ th order invariants for $V$.

Proof. We use induction: assume $\left[V^{(n-1)}, \Gamma\right]=\lambda \Gamma$ and suppose that $z^{(j)}$ is an invariant of $V$. To see that $z^{(j+1)}$ is an invariant of $V$ we use the quotient rule,

$$
\begin{aligned}
(\Gamma(u))^{2} V^{(n-1)}\left(z^{(j+1)}\right) & =V^{(n-1)} \circ \Gamma\left(z^{(j)}\right) \Gamma(u)-\Gamma\left(z^{(j)}\right) V^{(n-1)} \circ \Gamma(u) \\
& =\Gamma(u)\left[V^{(n-1)}, \Gamma\right]\left(z^{(j)}\right)-\Gamma\left(z^{(j)}\right)\left[V^{(n-1)}, \Gamma\right](u) \\
& =\Gamma(u) \lambda \Gamma\left(z^{(j)}\right)-\Gamma\left(z^{(j)}\right) \lambda \Gamma(u) \\
& =0
\end{aligned}
$$

hence $V^{(n-1)}\left(z^{(j)}\right)=0$ for $j=0, \ldots, n-2$.

To see the functional independence, we note how $\Gamma$ acting on a function effectively increases the order of derivatives which it depends on by one. Because $u$ is a zeroth order invariant with no $y^{\prime}$ dependence and $u$ and $z$ are functionally independent, $z$ must have nowhere zero $y^{\prime}$ dependence, and so $\Gamma(u)$ and $\Gamma(z)$ will have nowhere zero $y^{\prime \prime}$ dependence. From here the result follows by induction.

The condition that $\Gamma(z)$ and $\Gamma(u)$ are nowhere zero is often overlooked, yet failure of an invariant to satisfy this condition on an open region $U$ is not a problem, it is a bonus! We then have a first integral $z$ or $u$ on $U$, this is itself a reduced equation in the original coordinates.

Otherwise putting $\theta^{j}=d z^{(j)}-z^{(j+1)} d u$ for $j=1, \ldots, n-2$ and $\theta^{n-1}=$ $d z^{(n-1)}-\hat{f} d u$ we have $n-1$ independent 1 -forms which clearly satisfy $\left.\Gamma\right\lrcorner \theta^{j}=$ 0 and $V\lrcorner \theta^{j}=0$ and so

$$
V\lrcorner \Omega=\alpha \wedge \theta^{1} \wedge \theta^{2} \wedge \cdots \wedge \theta^{n-1}
$$

for some $\alpha \in \wedge^{0} J^{n-1}(\mathbf{R}, \mathbf{R})$. The $\theta^{j}$ for $j=1, \ldots, n-2$ form a natural contact structure and with the force form $\theta^{n-1}$ we have the reduced differential 
equation

$$
\frac{d^{n-1} z}{d u^{n-1}}=\hat{f}\left(u, z, z^{(1)}, \ldots, z^{(n-2)}\right) .
$$

The solutions of (6-2) are related to those of (5-1) by an auxiliary first order equation, but a better connection with our theory is that the $n-1$ first integrals of (6-2) are also first integrals of (5-1), leaving us short by one. If we have some function $s$ so that $V^{(n)}(s)$ is nowhere zero then the 1 -form $d s-\Gamma(s) d x$ is Frobenius integrable modulo the $n-1$ first integrals of (6-2) and thus gives us the last one.

We now consider the extension of this method for an arbitrary symmetry of (5-1), that is, we consider the case where $V$ is no longer a point symmetry, but we still have $[V, \Gamma]=\lambda \Gamma$ for some $\lambda$. Suppose we also know two invariants $u$ and $z$ of $V$, these need not be first order invariantsindeed this has no meaning as $V$ is not the prolongation of a base vectorfield. We may still construct other invariants as per Proposition 6.1, but we are no longer guaranteed their independence. As before we put $z^{(0)}=z$ and $z^{(j)}=$ $\Gamma\left(z^{(j-1)}\right) / \Gamma(u)$ but this time we stop when we have $u, z, z^{(1)}, \ldots, z^{(k-1)}$ functionally independent but $u, z, z^{(1)}, \ldots, z^{(k)}$ functionally dependent so that $z^{(k)}=g\left(u, z, z^{(1)}, \ldots, z^{(k-1)}\right)$. We then have the differential equation for $z(u)$

$$
\frac{d^{k} z}{d u^{k}}=g\left(u, z, z^{(1)}, \ldots, z^{(k-1)}\right)
$$

The $k$ first integrals of (6-3) are also first integrals of (5-1) as before. In the event that $k<n-1$, we need another invariant of $V$ to proceed. If we have the invariant $w$ of $V$ which is functionally independent of $u, z, z^{(1)}, \ldots, z^{(k-1)}$ then once again we put $w^{(0)}=w$ and $w^{(j)}=\Gamma\left(w^{(j-1)}\right) / \Gamma(u)$ and this time we stop when we have $u, w, w^{(1)}, \ldots, w^{(l-1)}, z, z^{(1)}, \ldots, z^{(k-1)}$ functionally independent but $u, w, w^{(1)}, \ldots, w^{(l-1)}, z, z^{(1)}, \ldots, z^{(k-1)}$ functionally dependent so that $w^{(l)}=h\left(u, w, w^{(1)}, \ldots, w^{(l-1)} ; z, z^{(1)}, \ldots, z^{(k-1)}\right)$. We then have the differential equation for $w(u)$,

$$
\frac{d^{l} w}{d u^{l}}=h\left(u, w, w^{(1)}, \ldots, w^{(l-1)} ; z, z^{(1)}, \ldots, z^{(k-1)}\right)
$$

Although this equation is not necessarily integrable in its own right, it is integrable once we have the solution of (6-3). In terms of the Pfaffians, the wedge of the $k$ Pfaffians is Frobenius integrable, while the $l$-form given by the wedge of the $l$ Pfaffians for (6-4) is integrable modulo the $k$ Pfaffians of (6-3). We continue in this way until we have $n-1$ Pfaffian forms in a chain of integrability. $X\lrcorner \Omega$ is then given by some multiple of the wedge of these $n-1$ Pfaffians, thus giving interpretation of $X\lrcorner \Omega=0$ as a reduced differential equation.

We have not restricted ourselves to one-dimensional equations: if $\Gamma$ is an $n$th order equation field for a system of $m$ equations, then this method works equally well; it might even be possible to reduce the system to a single $(m n-1)$ th order equation! It is also possible to perform such a reduction using canonical coordinates if $V$ is not a symmetry of $\Gamma$ but if the distribution $\operatorname{span}(\{\Gamma, V\})$ is integrable. Proposition 6.1 still holds, but we will omit the details of this.

If we look for canonical coordinates for a two dimensional solvable algebra of symmetries, say $V$ and $X$ with $[V, X]=\lambda V$ then we first find the invariants 
for $V$ as above, then look for the invariants of $X$. We can find coordinates $a, b, c^{j}$ so that $X(b)=1$ and $V(b)=X(a)=V\left(c^{j}\right)=X\left(c^{j}\right)=0$ and we can express (6-1) in terms of the invariants of both $V$ and $X$ with a reduction of order of 2 . If we consider a reduction by a full $m n$-dimensional solvable algebra and we find the invariant for this full algebra, it will be precisely the first integral $\gamma^{1}$ found using Proposition 5.3. Actually, the reduction process of $\S \mathrm{V}$ is ideally suited to finding the canonical coordinates, should this be desired.

We end this section with a discussion of the more formal traditional notion of a "reduced differential equation". In the literature this term usually refers to a differential equation on a quotient manifold. This reduction is performed on some initial equation by passing to the quotient of the original host manifold by a symmetry group action and using the corresponding differential invariants to produce a canonical coordinate representation of the equation. In our treatment of reduction of order these ideas are not in evidence and the reader may well ask in what sense our locally simple $p$-forms represent the usual reduced differential equations on the quotient. We made the claims in the example of §III that a multiple of the second order ordinary differential equation field $\Gamma$ always passes to the quotient and similarly that $X\lrcorner \Omega$ is a multiple of a form pulled back from the quotient. These two objects constitute the reduced differential equation of our theory. Importantly for our reduction of order algorithm we never need to pass to the quotient, the entire process can be carried out on the host manifold in an essentially coordinate free manner. However, for the sake of completeness, we give the following theorem.

Theorem 6.3. Let $\Gamma$ be an $n$th order equation field on an open $U \subseteq J^{n-1}\left(\mathbf{R}, \mathbf{R}^{m}\right)$ and $\Omega$ be a corresponding locally simple mn-form. Let $X$ be a symmetry of $\Gamma$ and hence of $\Omega$ (by Proposition 4.1), nowhere zero on $U$, and let $Q$ be the quotient of $U$ by the one-parameter group generated by $X$, with projection $\pi: U \rightarrow Q$. Then

(i) there exists $\mu \in \bigwedge^{0} U$ such that $\mu \Gamma$ passes to $Q$.

(ii) there exists $\sigma \in \Lambda^{0} U$ such that $\left.\sigma(X\lrcorner \Omega\right)=\pi^{*} \alpha$ where $\alpha \in \bigwedge^{m n-1} Q$.

(iii) $\operatorname{ker}(\alpha)=\operatorname{span}\left(\left\{\pi_{*}(\mu \Gamma)\right\}\right)$ and hence $\alpha$ is Frobenius integrable and so locally simple.

Proof. (i) A vectorfield $W$ passes to $Q$ if and only if $\mathscr{L}_{X} W=\rho X$ for some $\rho$, however we have $\mathscr{L}_{X} \Gamma=\lambda \Gamma$ by assumption. So we look for a multiple $\mu \Gamma$ of $\Gamma$ so that $\mathscr{L}_{X}(\mu \Gamma)=0$ (nontrivial multiples of $X$ are not possible). We find that $\mu$ must satisfy $X(\mu)+\mu \lambda=0$. If we attempt a solution $\mu=(\Gamma\lrcorner \omega)^{-1}$ for some appropriate 1 -form $\omega$ we find a necessary and sufficient condition for the existence of $\mu$ and hence $\omega$ is that $\Gamma\lrcorner \mathscr{L}_{X} \omega=0$, that is, $\mathscr{L}_{X} \omega$ is a constraint form for $\Gamma$. We can always find such forms: for example, if $g \in \wedge^{0} U$ is a differential invariant of $X$ so that $X(g)=0$, with $\Gamma(g) \neq 0$ on $U$ then $\omega=d g$ satisfies the requirement and so $\widehat{\Gamma}=\Gamma / \Gamma(g)$ passes to the quotient.

(ii) A form $\beta$ on $U$ is the pullback of a form $\alpha$ on $Q$ if and only if $X\lrcorner \beta=0$ and $X\lrcorner d \beta=0$, or equivalently, $\mathscr{L}_{h X} \beta=0$ for all $h \in \wedge^{0} U$. Now for $\beta=\sigma(X\lrcorner \Omega)$, the condition $X\lrcorner \beta=0$ is automatically satisfied and we need only be concerned with $X\lrcorner d(\sigma X\lrcorner \Omega)=0$ or $\left.\mathscr{L}_{X}(\sigma X\lrcorner \Omega\right)=0$.

Given that $\mathscr{L}_{X} \Omega=\bar{\lambda} \Omega$ by assumption, we find that a necessary and sufficient condition on $\sigma$ is $X(\sigma)+\bar{\lambda} \sigma=0$. Attempting a solution $\left.\left.\sigma=X\lrcorner Z_{1}\right\lrcorner \ldots\right\lrcorner Z_{m n-1}$ 
\lrcorner$\Omega$ for some appropriate vectorfields $Z_{1}, \ldots, Z_{m n-1}$ we find a sufficient condition for the existence of the $Z_{j}$ 's, and hence $\sigma$, is that

$$
\mathscr{L}_{X} Z_{j} \in \operatorname{span}\left(\left\{\Gamma, X, Z_{1}, \ldots, Z_{j-1}, Z_{j+1}, \ldots, Z_{m n-1}\right\}\right) .
$$

This condition is satisfied if $\mathscr{L}_{X} Z_{j}=0$ for each $Z_{j}$ so an obvious choice is $Z_{j}=\frac{\partial}{\partial v^{j}}$ where $X\left(v^{j}\right)=0$, and once again we recognise canonical coordinates.

(iii) To see that $\alpha$ has a one-dimensional kernel spanned by the projection of a multiple of $\Gamma$ to $Q$, consider $\widehat{W} \in \operatorname{ker} \alpha: \widehat{W}=\pi_{*} W$ for some $W$ on $U$ and so $0=\widehat{W}\lrcorner \alpha$ implies $\left.\left.\left.0=\pi^{*}(\widehat{W}\lrcorner \alpha\right)=\pi^{*}\left(\pi_{*} W\right\lrcorner \alpha\right)=W\right\lrcorner \pi^{*} \alpha$. Hence $W$ is in the kernel of $X\lrcorner \Omega$ which by assumption is $\operatorname{span}(\{X, \Gamma\})$. But this distribution passes to the quotient as $\operatorname{span}(\{\widehat{\Gamma}\})$ and hence $\alpha$ is Frobenius integrable and locally simple since its kernel is one dimensional.

It is clear from this theorem that the Pfaffian equation " $\alpha=0$ " on $Q$ is precisely the usual reduced differential equation arising from reduction by the symmetry $X$, and as a consequence we also identify the Pfaffian equation " $X\lrcorner \Omega=0$ " as the reduced differential equation (even though it has a twodimensional kernel).

\section{Conclusion}

We hope to have demonstrated to you that this geometric reduction of order technique is elegant, computationally simple and more powerful than the traditional approach. Apart from our original intention to generalise the integrating factor result our main efforts have been computational and there is no doubt that algorithmically the technique and its computer algebraic implementation are a radical improvement over the current ones. The theory, combined with a symmetry determination program such as DIMSYM [14] and an exterior calculus package such as EXCALC [15], makes child's play of many problems (see [6-8]). On the theory side, however, we have only scratched the surface as even a cursory glance at [1], for example, shows. We earnestly hope that our small contribution will awaken an interest in the pioneering work of É. Cartan amongst the many experts and practitioners in the group theory of differential equations.

Since the submission of this article, our attention has been brought to the work of others currently exploring some of the ideas contained here. BasarabHorwath [12] has worked on giving a geometrical description of Lie's generalisation of Jacobi's multiplier, and has given analogues of several results contained here such as Proposition 5.3 using changes of coordinates rather than modulo-closed forms. Duzhin and Lychagin [13] construct a basis of (almost basic) 1 -forms using Cartan's techniques $[4,5]$ to find first integrals, with some emphasis on distributions arising from PDE's. This generalisation is remarked upon here in $\S \mathrm{V}$ but is not emphasised as this only covers completely determined systems of PDE's.

We would Like to thank Peter Basarab-Horwath for providing references [9, 10,11 ]. In [9], Lie gives $\omega^{1}$ and $\omega^{2}$ of Corollary 3.4 where

$$
\Gamma=\frac{\partial}{\partial x}+p \frac{\partial}{\partial y}+f(x, y, p), \quad V=\tau_{V} \frac{\partial}{\partial x}+\xi_{V} \frac{\partial}{\partial y}+\xi_{V}^{(1)} \frac{\partial}{\partial p},
$$


as

$$
W=\tau_{W} \frac{\partial}{\partial x}+\xi_{W} \frac{\partial}{\partial y}+\xi_{W}^{(1)} \frac{\partial}{\partial p}
$$

$$
\begin{gathered}
\Delta=\left|\begin{array}{ccc}
1 & p & f(x, y, p) \\
\tau_{V} & \xi_{V} & \xi_{V}^{(1)} \\
\tau_{W} & \xi_{W} & \xi_{W}^{(1)}
\end{array}\right|, \quad \omega^{1}=\frac{1}{\Delta}\left|\begin{array}{ccc}
d x & d y & d p \\
1 & p & f(x, y, p) \\
\tau_{W} & \xi_{W} & \xi_{W}^{(1)}
\end{array}\right|, \\
\omega^{2}=-\frac{1}{\Delta}\left|\begin{array}{ccc}
d x & d y & d p \\
1 & p & f(x, y, p) \\
\tau_{V} & \xi_{V} & \xi_{V}^{(1)}
\end{array}\right| .
\end{gathered}
$$

He also goes on to give the obvious generalisation for a pfaffian equation in four variables with three symmetries. It is most unfortunate that this work has not been translated into English, as both Lie's and Cartan's works contain many gems yet to be realised. Cohen [10, pp. 124-174] and Dickson [11, pp. 341-342, 354-356] give this result without proof, with only Dickson referencing Lie [9, pp. 453-456].

\section{REFERENCES}

1. P. Olver, Applications of Lie groups to differential equations, Springer-Verlag, New York, 1986.

2. I. Vaisman, Cohomology and differential forms, Marcel Dekker, New York, 1973.

3. M. Crampin and F. Pirani, Applicable differential geometry, Cambridge Univ. Press, Cambridge, 1987.

4. É. Cartan, Leçons sur les invariants intégreaux, Hermann, Paris, 1922.

5. —_ Les systèms différentiels extérieurs et leurs applications géométriques, Hermann, Paris, 1945.

6. J. Sherring, An EXCALC program for integration modulo closed one forms, La Trobe University Mathematics Department Research Report, 1990.

7. S. Godfrey and G. Prince, A canonical reduction of order for the Kepler problem, J. Phys. A 24 (1991), 5465-5475.

8. G. Prince and J. Sherring, An EXCALC package for tangent bundle calculations with second order equations, La Trobe University Mathematics Department Research Report, 1990.

9. S. Lie, Vorlesungen über Differentialgleichungen mit bekannten infinitesimalen Transformationen, Leipzig, Teubner, 1891.

10. A. Cohen, An introduction to the Lie theory of one-parameter groups, Stechert, New York, 1911.

11. L. E. Dickson, Differential equations from the group standpoint, Ann. of Math. 25 (1924), 287-378.

12. P. Basarab-Horwath, Integrability by quadrature for systems of involutive vector fields, Report, Department of Mathematics, University of Linköping, Sweden, 1990.

13. S. V. Duzhin and V. V. Lychagin, Symmetries of distributions and quadrature of ordinary differential equations, Report, Program Systems Institute of the USSR Academy of Sciences, 1991.

14. J. Sherring, DIMSYM, a REDUCE program for automatic calculation of symmetries of differential equations and distributions of vectorfields, La Trobe University Mathematics Department Research Report, 1992.

15. E. Schruefer, EXCALC user's manual: A system for doing calculations in the calculus of modern differential geometry, 1986.

Department of Mathematics, la Trobe University, Bundoora, Victoria 3083, AusTRALIA

E-mail address, James Sherring: matjs@lure.latrobe.edu.au

E-mail address, Geoff Prince: matgp@lure.latrobe.edu.au 